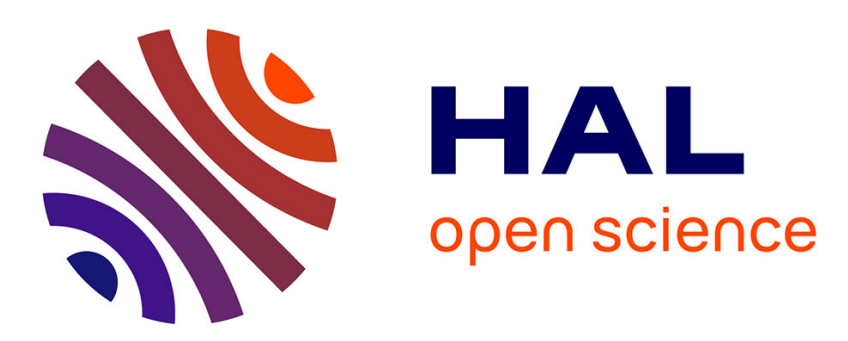

\title{
Tactile Fixations: A Behavioral Marker on How People with Visual Impairments Explore Raised-line Graphics
}

Kaixing Zhao, Sandra Bardot, Marcos Serrano, Mathieu Simonnet, Bernard Oriola, Christophe Jouffrais

\section{To cite this version:}

Kaixing Zhao, Sandra Bardot, Marcos Serrano, Mathieu Simonnet, Bernard Oriola, et al.. Tactile Fixations: A Behavioral Marker on How People with Visual Impairments Explore Raised-line Graphics. CHI 2021: Conference on Human Factors in Computing Systems, ACM, May 2021, Yokohama, Japan. 10.1145/3411764.3445578 . hal-03136004

\section{HAL Id: hal-03136004 https://hal.science/hal-03136004}

Submitted on 9 Feb 2021

HAL is a multi-disciplinary open access archive for the deposit and dissemination of scientific research documents, whether they are published or not. The documents may come from teaching and research institutions in France or abroad, or from public or private research centers.
L'archive ouverte pluridisciplinaire HAL, est destinée au dépôt et à la diffusion de documents scientifiques de niveau recherche, publiés ou non, émanant des établissements d'enseignement et de recherche français ou étrangers, des laboratoires publics ou privés. 


\section{Tactile Fixations: A Behavioral Marker on How People with Visual Impairments Explore Raised-line Graphics}

KAIXING, ZHAO

IRIT, University of Toulouse, France

SANDRA, BARDOT

University of Manitoba, Canada

MARCOS, SERRANO

IRIT, University of Toulouse, France

MATHIEU, SIMONNET

LEGO, IMT Atlantique, France

BERNARD, ORIOLA

IRIT, CNRS, France

CHRISTOPHE, JOUFFRAIS

IPAL, CNRS, Singapore

Raised-line graphics are tactile documents made for people with visual impairments (VI). Their exploration relies on a complex two-handed behavior. To better understand the cognitive processes underlying this exploration, we proposed a new method based on "tactile fixations". A tactile fixation occurs when a finger is stationary within a specific spatial and temporal window. It is known that stationary fingers play an active role when exploring tactile graphics, but they have never been defined or studied before. In this study, we first defined the concept of tactile fixation, then we conducted a behavioral study with ten participants with $\mathrm{VI}$ in order to assess the role of tactile fixations under different conditions. The results show that tactile fixations vary according to different factors such as the graphic type as well as the involved hand and the aim of the exploration.

CCS CONCEPTS • Human-centered computing Accessibility

Additional Keywords and Phrases: Tactile Fixations, Raised-line graphic, Visual impairment

ACM Reference Format: 


\section{INTRODUCTION}

Raised-line graphics are commonly used in education centers for people with visual impairments (VI) to provide access to mathematical graphs, maps, drawings, etc. These graphics are often printed on Swell Touch Paper and allow people with VI to explore the lines (raised by the printer) with their fingers. The design of such graphics include many elements such as legends, textures, symbols, dots, lines, etc. [12] and the complexity of the graphic depends on the number of these elements. The design process relies on the experience of the document maker and depends on many factors such as the perceptual and cognitive capacities of the end-user, the type and the aim of the graphic, etc. Therefore, there is a need to better understand how people with VI explore such graphics. This knowledge would help to design better tactile graphics but would also help to design better interactive graphics (i.e. talking tactile graphics, see [7] [30])

When exploring a raised-line graphic, cognitive exploration strategies may differ across users [1] [21] [25] [46]. The basic purpose of the cognitive exploration strategies is to find out what information is encoded in the graphic and to elaborate and memorize a functional mental representation of the graphic [22]. In fact, hand movements can reveal underlying strategies [19] [42]. For example, Wijnthes et al. [46] identified three categories of hand movements based on the use of one hand only, both hands alternately or both hands simultaneously. All these studies revealed that the fingers are not always moving and may perform different actions during the exploration. For instance, it has been observed that the hands can be stationary during the exploration. But, little is known about how people with VI use their two hands to explore these graphics, and there is a lack of measures to evaluate these explorations.

In this work, we focused on the users' finger movements by introducing a behavioral marker called 'tactile fixation". A tactile fixation occurs when a finger is motionless within a spatial and temporal window. When people with VI explore a raised-line graphic, their fingers often stop over specific elements of the graphics. Identifying tactile fixations can provide valuable information on the salient areas of the graphics and on the exploration strategies of the users. Therefore, in this paper, we address different research questions on how to identify tactile fixations and on the role of tactile fixations during the exploration of raised-line graphics.

To answer these questions, we conducted a behavioral study with 10 people with VI who performed tactile exploration tasks on different types of raised-line graphics and with different instructions. We logged the participants' finger movements and we identified tactile fixations from the recorded finger paths with a fixation detection algorithm based on previous research on eye movements. The statistical analyses show that there are tactile fixations of different durations, which are involved in different one-handed or two-handed behavioral patterns. Finally, we illustrate these different behavioral patterns and discuss the interest of this new approach on the design of raised-line graphics as well as on the design of non-visual interfaces or/and interaction techniques.

In summary, our contributions are: 1) the definition of the concept of "tactile fixation" which is a behavioral marker on how people with VI explore raised-line graphics; 2) a method (with an algorithm) to detect "tactile fixations"; 3 ) the analysis of tactile fixations in terms of perception and comprehension of tactile graphics using a set of hand movement data.

\section{RELATED WORK}


Most of previous work on raised-line graphics exploration has focused on how people with VI explore tactile graphics, including the analysis of cognitive strategies and the identification rates of the graphics. In this section, we provide a presentation of these findings.

\subsection{Tactile exploration of graphics}

Hand-based tactile exploration [34] is an important way to retrieve information, especially for people with VI. Previous research has started to investigate how people with VI use their hands to explore raised-line graphics [1] [36] [42] [46]. For example, Symmons et al. [42] described hand movements of blindfolded sighted participants during the exploration of raised-line graphics. They showed that most subjects use their index finger(s), either alone or in combination with other fingers. Participants generally explored the left part of the graphic with their left hand and the right part with their right hand. In addition, Bardot et al. [1] proposed a study with five types of raised-line graphics and compared the exploration of blindfolded people and people with VI. The results showed that people with VI used mainly the left hand to explore a graphic compared to blindfolded people. Finally, Wijntjes et al. [46] classified hand movements into three categories: a) use of one hand (the other hand is outside of the graphic) to perceive graphical elements; b) alternate use of both hands (while one hand explores, the other hand is motionless on the graphic); c) simultaneous use of both hands (both hands explore at the same time). The authors showed that participants explored with both hands in more than $83 \%$ of the exploration time, which significantly increased the identification of raised-line graphics. Altogether, it suggests that using both hands during a tactile exploration is more effective than using a single hand.

\subsection{Recognition of tactile graphics}

The field of psychology has widely studied whether visual experience is required for the identification or recognition of raised-line graphics [20] [21] [27] [31] [33] [36] [45]. Generally, these studies focused on raisedline graphics of common objects (usually from Snodgrass et al. [41]) or patterns (i.e. specific graphic combinations [31] [47]) which participants had to recognize. The studies were based on comparative studies involving people with different visual capacities, such as early blind, late blind and sighted people, which showed contradictory results. Some studies showed that sighted people had better recognition rates than people with VI [35] [45]; while others found opposite results [21]. Interestingly, Lebaz et al. [31] used non-significant graphics instead of common objects and found that recognition depends on the participant's profile (i.e. spatial, visuospatial or kinesthetic memory). Nevertheless, these studies still mainly focus on the identification or recognition of the raised-line graphics and have never tried to further investigate participants' cognitive exploration strategies during the exploration process. Therefore, although we have the basic knowledge that people with $\mathrm{VI}$ are able to identify raised-line graphics, it is still not clear how they rely on tactile exploration to understand tactile graphics.

\subsection{Cognitive exploration strategies without vision}

Prior works on tactile exploration without vision have shown different cognitive exploration strategies when people with $\mathrm{VI}$ discover and memorize spatial graphics, but none of them investigated this research question based on tactile fixations. Although it is a challenge to link exploration behaviors (i.e. what one can observe) with the goals (i.e. what the user intends to do) [32], the observation of the exploratory movements on the spatial layout can provide information on the underlying user's cognitive strategy. It might not be exhaustive, but to 
date, different studies converge towards the four following main cognitive exploration strategies to build spatial mental maps [44] without vision.

- The Grid strategy [22]: users do systematic horizontal and vertical movements to locate all the elements depicted in the graphic.

- The Perimeter strategy [22]: users follow the outline(s) of the graphic. With this strategy the user aims to identify the main element(s) in the graphics based on their shape(s).

- The Cyclic strategy [15]. The cyclic strategy consists in browsing a series of elements in the graphics and finally coming back to the first element. The aim of this strategy is to memorize the relative location of different elements in the graphics.

- $\quad$ The Reference point strategy [43]: it consists in touching different elements of the graphics located around one element called the reference point. This "star-like" strategy aims to understand the relation between graphical elements located around this landmark. Relying on this strategy, users intend to encode a reference that is efficient in building a mental image of the graphic.

In addition to these studies in psychology, recent studies on interactive 3D printed maps [16] [24] and digital interactive graphics [2] [10] [17] [18] [26] studied exploration behaviors involving one or two hands. For instance, Guerreiro et al. [18] observed various two-handed strategies including symmetrical hand movements when exploring the graphic. Interestingly, new technologies make it easier to track finger movements and can improve the research in the domain by providing new methods [6] [29].

\subsection{Summary}

In summary, the related work on the exploration of tactile graphics by people with VI shows that the main focus of recent studies is more on the demographic factors that impact the identification and the recognition rates of graphics but maybe not enough on the exploration movements and the cognitive strategies behind them. Although Wijntjes et al. [46] have shown that hand movements on tactile drawings can be instructed to three categories (one-handed, two-handed alternately and two-handed simultaneously) and also observed that the two hands are moving during $83 \%$ of the exploration time, there is still a lack of research focusing on the finger movements (including the stops) done by people with VI when exploring tactile graphics. In fact, there is a need to propose a method to better describe hand movements and subsequently understand cognitive strategies behind them. In our study, we have developed a method for detecting tactile fixations inspired by ocular fixations. We have also observed three types of two-handed patterns based on tactile fixations: Anchor Point, Switching Fingers and Chaining Hands.

\section{TACTILE FIXATIONS: CONCEPT AND RESEARCH QUESTIONS}

In this section, we first introduce the concept of Tactile Fixations by drawing a parallel with eye fixations. Next, we introduce our research questions.

\subsection{Concept}

To introduce the concept of tactile fixations, we did a comparison with eye fixations although both are inherently different. When searching for a specific visual information, human eyes are either in movement (saccades) or still (fixations). The human eye can perceive information during the fixations, which are usually short, between 200-300 ms [4] [5] [9] [40]. Researchers often analyze eye movements to assess the user's attention and better 
understand how images, texts or web pages are perceived [9]. Thus, the study of eye fixations informs on the attention devoted to the different regions of the image and the saliency of the visual content [5].

Previous research on tactile exploration (see related work) has already identified behaviors where the exploring hand stays still for a certain time during the exploration. We made the hypothesis that the eye fixation paradigm can apply to the tactile exploration of tactile graphics. More precisely, we suggest that tactile fixations are single behaviors that are associated and organized to set up different cognitive strategies. During fixations, fingers can detect salient elements of the graphic, but they can also be anchor points used to better understand the relationships between graphical elements, and hence the signification of the whole graphic. The main difference between eye and tactile fixations are related to the fact that: i) the hands can move along an outline and hence do not always jump from one element to the other; ii) the two hands are more independent than the two eyes, allowing complex two-handed strategies.

\subsection{Research questions}

"Tactile fixations" have been observed in previous work on tactile exploration [42] [46]. However, we know little about when and why they occur. To drive our investigation, we identified a set of research questions related to tactile fixations:

1) How to detect tactile fixations?

2) Do participants perform tactile fixations with both hands?

3) Do tactile fixations vary according to the type of graphic?

4) Do tactile fixations vary according to the exploration task? The hand movement may not be the same if the participant is doing a non-guided vs. guided exploration (i.e. to find the response to a question about the graphic).

5) Can we relate tactile fixations to exploration patterns?

To investigate these research questions, we did a behavioral study to collect finger movements related to two-handed exploration of different types of graphics explored with different instructions.

\section{BEHAVIORAL STUDY}

\subsection{Participants}

We recruited 10 participants with visual impairments (4 females, 6 males) with an average age of 52.3 (SD = 13) from a local special education center. Among them, eight were legally blind (according to the definition of U.S. Social Security Administration (SSA), people are considered as legally blind if the better eye - when using a corrective lens - has a central visual acuity of 20/200 or lower, or if the field of vision is smaller than 20 degrees.) and two had limited residual vision (among the two participants with residual vision, one can only perceive light and shadows and the other can only perceive light and contrast. None of them can use its residual vision to understand drawings, and both were taught to explore tactile drawings. Although not mandatory, we blindfolded them to make sure they did not use any visual cues to identify the drawing without exploring it.). On average, blind participants lost sight at 10 years old $(S D=17)$. All the participants were right-handed and all of them read Braille with their left hand. The details of the participants are presented in Table 1.

We conducted an interview to assess their level of expertise before starting data collection. Five participants reported that they had never received any training on raised-line graphic exploration. Five of them received 
lessons on tactile exploration. Only one person reported using raised-line graphics daily. The other participants mentioned that they do not use them regularly: five participants do not have any raised-line graphics at home but can occasionally use them, and four participants do not have the need nor the opportunity to use raisedline graphics.

Table 1. Details of the participants

\begin{tabular}{lllllcccc}
\hline ID & Gender & Age & Degree VI & Residual & $\begin{array}{c}\text { Onset age } \\
\text { of VI }\end{array}$ & $\begin{array}{c}\text { Age } \\
\text { learning } \\
\text { Braille }\end{array}$ & $\begin{array}{c}\text { Braille } \\
\text { expertise }(1-\end{array}$ & $\begin{array}{c}\text { Dominant } \\
\text { hand }\end{array}$ \\
\hline P1 & M & 45 & Blind & No & 0 & 5 & 4 & Right \\
\hline P2 & F & 61 & $\begin{array}{l}\text { Visually } \\
\text { impaired }\end{array}$ & $\begin{array}{l}\text { Light } \& \\
\text { Contrast }\end{array}$ & 15 & 18 & 3 & Right \\
\hline P3 & M & 62 & Blind & No & 3 & 5 & 6 & Right \\
\hline P4 & M & 68 & Blind & No & 2 & 5 & 5 & Right \\
\hline P5 & F & 37 & Blind & No & 2 & 5 & 6 & Right \\
\hline P6 & M & 46 & Visually & Light \& & 18 & 18 & 1 & Right \\
\hline P7 & M & 45 & Blind & No & 0 & 6 & 7 & Right \\
\hline P8 & F & 63 & Blind & No & 57 & 61 & 1 & Right \\
\hline P9 & F & 48 & Blind & No & 5 & 9 & 6 & Right \\
\hline P10 & M & 48 & Bind & No & 5 & 12 & 4 & Right \\
\hline
\end{tabular}

\subsection{Raised-line graphics}

In our study, we introduced five different types of raised-line graphics including 2D Drawings, Drawings with Perspective, Mathematical Graphs, Geographic Maps, and Neighborhood-Building Maps. All the graphics were made with the assistance of a tactile document maker with 15 years of expertise and working in a special education center for people with VI, as well as with a blind people frequently using tactile documents. The graphics were printed on A3 sheets of Swell Touch Paper. The detailed information of these graphics are as follows:

a) 2D Drawings. We selected 2D drawings from a set of 260 images designed in [41], which was created for being used in psychology experiments. We have selected graphics representing common animals only (Figure 1-A) in order to have graphics from a single family that users know.

b) Drawings with Perspective. The drawings with perspectives were chosen from the same image set [41]. We separated 2D drawings from drawings that include perspectives because the perspective is a visual convention used to give solid objects drawn on a flat surface the appearance of depth and distance. Perspective does not correspond to any tactile experience but introduces additional difficulties during tactile exploration [12] [14]. In this category, we have selected tools of transportation (Figure 1-B) in order to get graphics from a single family that users know (similar to 2D drawings). It must be noted that, according to the tactile document maker, the details represented in our 2D drawings and drawings with perspective are not adapted for tactile exploration by people with VI due 
to the occlusions and perspectives represented in the drawings. However, we included these drawings because similar ones have been used in different studies on tactile exploration in psychology [28] [36], especially in [31] that compared the accuracy and response time in identifying raised-line drawings.

c) Mathematical Graphs. We have designed this set in accordance with [37]. Here, two types of mathematical graphs were included: line graphs that display information as a series of points linked by segments, and histograms with vertical or horizontal bars (Figure 1-C). For both types of graphs, the $x$ - and $y$-axis were drawn with thick lines. We also added dashed lines to represent the main graduations on each axis. For line graphs, we used two different line styles (solid line and tight dotted line) to represent the series of data. For the histograms, we used different textures for different series of bars. The legend was added at the bottom right of the graphic.

d) Geographic Maps. We have designed two types of geographic maps showing either the different regions of a country or the different cities of a country (Figure 1-D) [13]. The borders between neighboring countries were drawn with solid lines and the borders between regions were drawn with dotted lines. All the cities were represented by solid circles [12] and each city or region was associated with a Braille number for simplicity. The sea was represented by a specific texture and the caption was added at the bottom right of the graphic which includes two columns: the symbols in the left column and the Braille legend in the right column.

e) Neighborhood-Building Maps (Figure 1-E). In the neighborhood maps, the streets was represented by solid lines (sidewalks and street width do not appear), as proposed in [3]. For additional elements on the map, we have used both solid dots or solid/empty triangles which are symbols recommended in [38]. The caption was added at the bottom right of the graphic. In the building maps, the walls are represented by solid lines and the gaps correspond to the doors. Each room is identified by a Braille number.

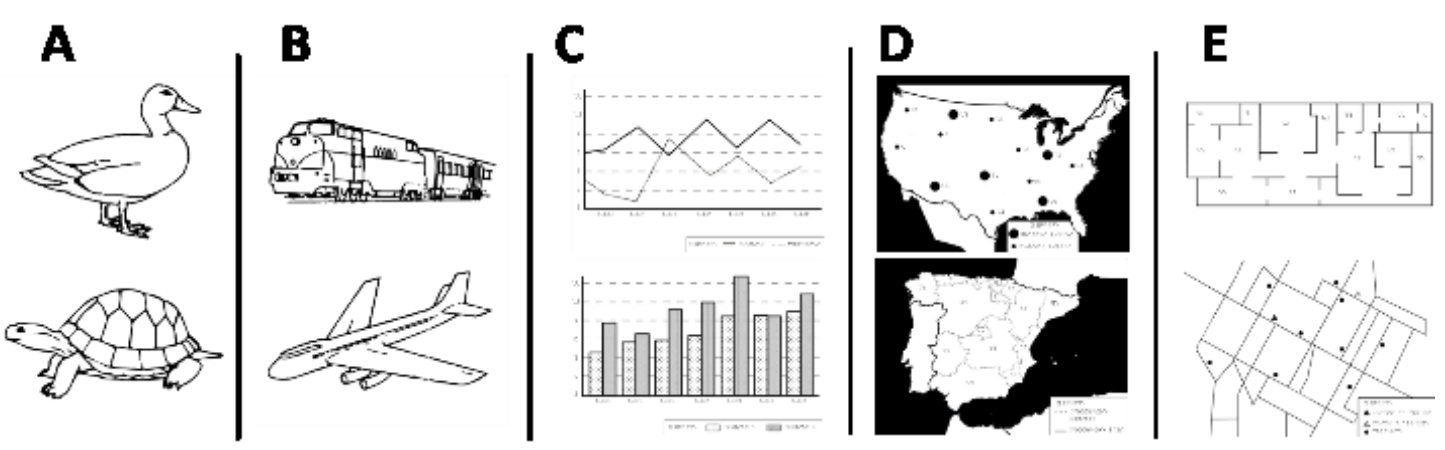

Figure 1. Example of raised-line graphics used for behavioral study. A: 2D Drawings; B: Drawings with Perspective; C: Mathematical Graphs; D: Geographic Maps; and E: Neighborhood-Building Maps.

\subsection{Experimental setup}

During the experiment, participants were comfortably sitting in front of the raised-line graphic placed on a table. A GoPro Hero4 camera $(60 \mathrm{~Hz})$ was attached above the graphics. We painted the left and right index fingernails of the participants with red and yellow nail varnish respectively to track them using computer vision algorithms 
(see section 4.5). The explanations about the experiment and instructions were read verbally to get participants' consent.

\subsection{Task and Procedure}

The instruction can strongly influence how people explore tactile graphics [1]. Hence, we designed three types of tasks based on different instructions. Among the three tasks, the first two tasks focus on image identification while the third focuses on image comprehension.

1) Free exploration (Free): the participant freely explores the graphic for up to 60 seconds until eventual identification.

2) Exploration with context (Context): before the exploration, the experimenter mentions the graphic type. The participant then freely explores the graphic for up to 60 seconds until eventual identification.

3) Purposeful exploration (Purpose): Before the exploration, the experimenter mentions the graphic type and provides an instruction related to the graphic elements. The participant explores the graphic for up to 90 seconds to answer the question.

The questions differed according to the type of graphic. For 2D drawings or drawings with perspectives, they were forced choice questions with two levels (e.g. "is it a <animal $1>$ or a <animal 2>?"). We chose two similar animals not to make the task too easy. For the mathematical graphs, the questions were about comparing data (e.g. "Are purchases more important than sales in <year 1> or <year 2>?"). For geographical maps, questions were about distance or size (e.g. "Is <city $1>$ closer to <city $2>$ or <city $3>$ ?" or "Is < region $1>$ larger than <region $2>$ ?"). For neighborhood maps, the questions focused on path departure and arrival ("Between departure and arrival points on that path, which one is the closest to a grocery?"). Finally, for building maps, the questions were about comparing different rooms (e.g. "Is <room 1> closer to <room 2> or <room 3>?").

The experiment followed a within-subject design with two factors: the type of graphics (2D Drawings, Drawings with Perspective, Mathematical Graphs, Geographical Maps and Neighborhood-Building Maps) and the task (Free, Context, and Purpose). The study was divided into three blocks corresponding to each of the three tasks. Each block was composed of 10 trials with two raised-line graphics of each type. Within each block, the order of the trials (graphics) was randomized. The order of the blocks was always the same for all the participants: 1) Free, 2) Context, and 3) Purpose. The maximum duration for each trial was 60 seconds (Free and Context tasks) or 90 seconds (Purpose). During the experiment, users were free to take a break between blocks.

\subsection{Methods}

In total, each participant explored 30 raised-line graphics. We collected: 3 (tasks) * 5 (types of graphics) * 2 (repetitions) $* 10$ (participants) $=300$ trials.

\subsubsection{Finger Tracking Algorithm}

In order to detect tactile fixations, we tracked the participants' finger movements by leveraging color tracking algorithms. Our tracking algorithm is based on a series of image processing techniques that enable us to detect predefined colored patches (color on nails) in each video frame. More precisely, the main steps of our method are: 1) Gaussian Blurring with the aim of reducing image noise and enhancing image structures; 2) HSV color space transformation. HSV (Hue, Saturation, Value) color space is an alternative representation of the RGB 
color model. It is often used for object detection and tracking in computer vision; 3) Colored patch tracking in each video frame; 4) Corrosion operation to decrease the size of the detected colored patches, which can be used to discard small and meaningless targets; 5) Region expansion aiming at increasing the size of meaningful colored patches, so that neighboring colored points are merged.

We applied our finger tracking algorithm on 296 videos (4 videos were discarded because the participants' head was accidentally recorded). Duration of the videos differs according to the participants and tasks. They are between 12 and 90 seconds long, and the average duration is 54.8 seconds. We calculated the hit rate of our finger tracking algorithm which is the ratio between the number of frames with finger detection and the total number of frames in all the trials. To make sure we can obtain a high hit rate, we conducted a data cleaning process: we developed a Python program to play each video frame by frame. For each frame without finger tracking, we manually verified the reason. If the missing tracking occurred when the finger was out of the image or when another finger was masking the colored patch, we moved to the next frame. If the absence of tracking was due to the performance of the finger tracking algorithm, we manually added a tag in the video. Finally, the hit rates for the left and right index fingers were respectively $98.0 \%$ and $97.3 \%$. The missing detections were due to the reasons presented earlier.

\subsubsection{Detection of Tactile Fixations}

As said earlier, we define a tactile fixation as the exploring finger being stationary within a given spatial and temporal window. Due to the technical similarity between eye fixations and tactile fixations, we applied the Dispersion-Threshold Identification (I-DT) algorithm that was initially used for detecting ocular fixations [40]. The I-DT algorithm is relatively simple to use compared to other algorithms that generally require specific data. For I-DT, only two parameters are needed: maximal spatial dispersion and minimal fixation duration. Both parameters are important since they define the tolerance of the detection algorithm. Increasing dispersion or decreasing duration leads to detecting more fixations. It is important to note two observations: i) hand movements are more continuous than eye movements and sometimes jump from one element to the other (saccade like movements). Hence, it is possible to detect very short tactile fixations if the fixation duration threshold is too low; ii) a finger is not rigorously static during a fixation. In fact, it is slightly moving to retrieve information about the underlying element. Hence, the spatial dispersion threshold must tolerate these small movements.

Therefore, an important question concerning the use of I-DT algorithm is the value of the two parameters. The threshold values of the eye fixations (usually between 20-50 pixels for the dispersion and 200-300 ms for the duration) are not applicable for tactile fixations because the hand movements are less accurate and slower than eye movements. The minimum fixation duration and the maximum spatial dispersion should be greater than for eye fixations. Considering our setting, we used 50 pixels for dispersion, which corresponds to $14 \mathrm{~mm}$ (the correspondence between 50 pixels and $14 \mathrm{~mm}$ is specific to the conditions of our study. It depends on the distance between the camera and the surface and on the camera lens being used too). The value of 50 pixels (e.g. $14 \mathrm{~mm}$ ) is large enough for detecting a tactile fixation since the average dimension of an index finger is 9 $\mathrm{mm}$ width and $16 \mathrm{~mm}$ length [36]. We used $500 \mathrm{~ms}$ as the minimum fixation duration because it discards very brief fixations during exploration. Using a shorter duration would result in detecting a very large number of events that are not relevant. 


\subsubsection{Statistical tests}

For all the measured variables, we firstly used a Shapiro-Wilk or Anderson-Darling (if the number of data is more than 5000) test to determine if their distribution was normal. If not, we tried to transform the data to get a normal distribution with a Box-Cox transformation [39] and then conducted an ANOVA. For the data that could not be transformed, since our study followed a within-subject design, we conducted a Friedman test which is specifically intended for non-parametric data.

\subsection{Results}

In this section, we first report the identification rates (i.e. how well participants completed each task). Then we detail our findings on the temporal distribution of tactile fixations. We then conduct a systematic analysis of tactile fixations on the graphics inspired by different methods indicated in [8] and we describe three recurrent movement patterns involving one or two hands.

\subsubsection{Identification rate and correct answers rate}

We calculated the graphic identification rate for the Free and Context tasks, as well as the percentage of correct answers for the Purpose task. Participants had an identification rate of $32.9 \%(\mathrm{Cl}[22.1 \%, 43.7 \%])$ in the Free task and $60 \%(\mathrm{Cl}[49.0 \%, 70.9 \%])$ in the Context task. The percentage of correct answers in the Purpose task was $80 \%(\mathrm{Cl}[67.7 \%, 92.3 \%])$.

\subsubsection{Tactile Fixations}

To get comparable results for the two hands, we conducted a preprocessing step to discard fixations detected on legends. The rationale for excluding these fixations comes from [1], which indicated that people with VI more often use their left hand to read Braille (especially legends). Therefore, we ignored these fixations to remove the effect of legends when comparing the two hands. In total, we detected 13209 fixations for all users (instead of 14975 fixations before the preprocessing phase).

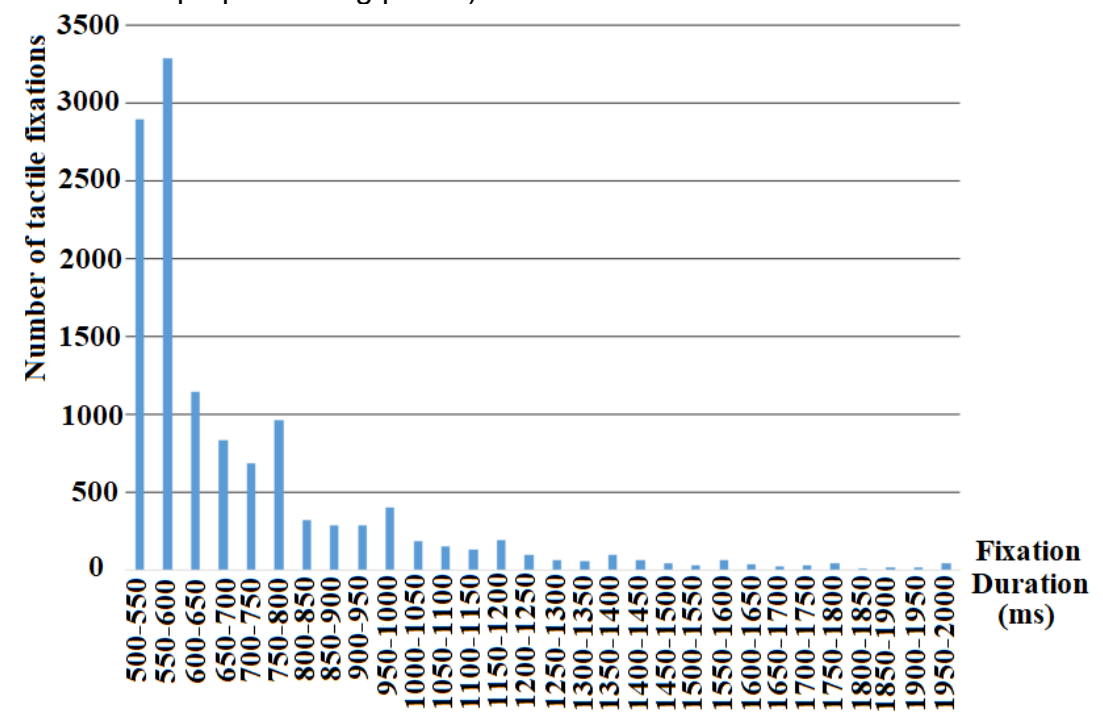

Figure 2. Distribution of tactile fixations according to fixation duration. 
We generated a distribution of the tactile fixations (see Figure 2). From this figure and the original data, we can make four observations: 1) The durations of tactile fixations vary from $500 \mathrm{~ms}$ to $48 \mathrm{~s}$ (very long fixations correspond to "forgotten hands", see discussion); 2) The number of tactile fixations decreases when the fixation duration increases with a lognormal distribution; 3 ) $84.3 \%$ of the tactile fixations are less than 1 -second-long; 4) There is a peak of the durations between 550 and $600 \mathrm{~ms}$.

\subsubsection{Number of Tactile Fixations}

We analyzed the number of fixations according to three variables: Hand, Graphic Type and Task. The ShapiroWilk test showed that the number of tactile fixations does not follow a normal distribution $(w=.98, p<.001)$. We transformed the distribution to normal using Box-Cox (after the transformation, the distribution was normal with $p=.189$ ). The ANOVA on the transformed data showed a significant difference between the numbers of tactile fixations according to both Hand $(F=11.94, p<.001)$ and Graphic Type $(F=7.89, p<.001)$, but not to the Task $(F=.13, p=.878)$. There is also an interaction between Task and Graphic Type $(F=3.33, p<.001)$.

The average number of tactile fixations was 24.2 on the left finger and 20.4 on the right finger (see Figure 3a). Although the tactile fixations on the legend areas have been discarded, we still observe more fixations with the left finger than the right finger.

The Tukey post-hoc test showed a significant difference between 2D Drawings and the four other types of graphic (Drawing with Perspective: $p=<.01$, Geographic Map: $p<.05$, Mathematical Graph: $p<.001$, Neighborhood-Building Map: $p<.01)$. The average number of fixations was smaller for 2D Drawings (17.8) than for Drawings with Perspective (22.9), Geographic Maps (22.4), Mathematical Graphs (25.2) and Neighborhood-Building Maps (23.3), as illustrated in Figure 3-b.

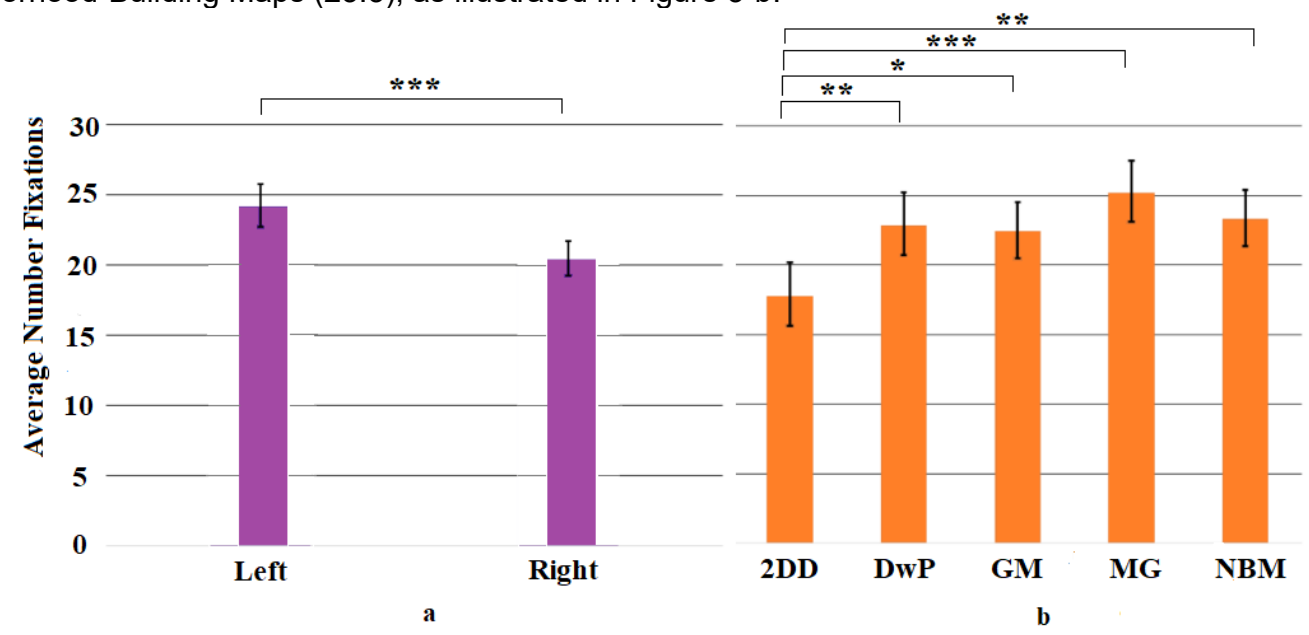

Figure 3. a. Average number of left and right tactile fixations, b. Average number of tactile fixations according to the type of graphic. $(95 \% \mathrm{Cl})$. 2DD: 2D Drawings; DwP: Drawings with Perspectives; GM: Geographic Maps; MG: Mathematical Graphs; NBM: Neighborhood Building Maps.

Concerning the interaction between Task and Graphic Type $(p=0.012$, see Figure 4), the Tukey post-hoc test shows that there is a significant difference between the number of fixations on $2 \mathrm{D}$ Drawings and the number 
of fixations on Drawings with Perspective $(p<.05)$, Mathematical Graphs $(p<.001)$ and Neighborhood-Building Maps $(p<.001)$ for the Purpose task. We did not find such difference between 2D Drawings and the other graphics for the Free and Context tasks (see Figure 4).

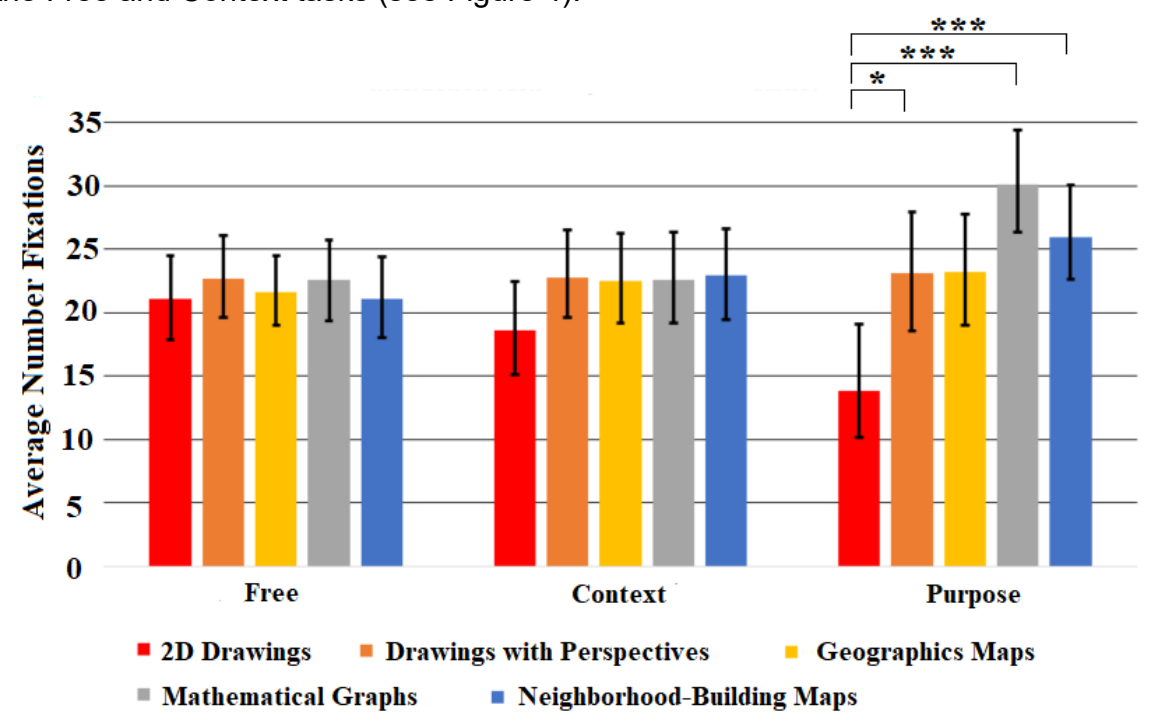

Figure 4. Number of fixations according to the Task and Graphic Type. (95\% Cl)

\subsubsection{Duration of Tactile Fixations}

The Anderson-Darling test (instead of Shapiro Wilk test because we had more than 5000 samples) shows that the fixation durations do not follow a normal distribution $(A=2890.2, p<.001)$. Since the distribution cannot be transformed to normal, we used a Friedman test which shows that there is a significant difference of fixation durations according to Hand $(p<.01)$, Graphic Type $(p<.001)$ and Task $(p<.001)$. There is also an interaction between Task and Graphic Type $(p<.001)$.

The overall average fixation duration was $897 \mathrm{~ms}$. As shown by the Friedman, it is significantly shorter with the left hand ( $835 \mathrm{~ms}$ ) than with the right hand (970 ms; see Figure 5-a).
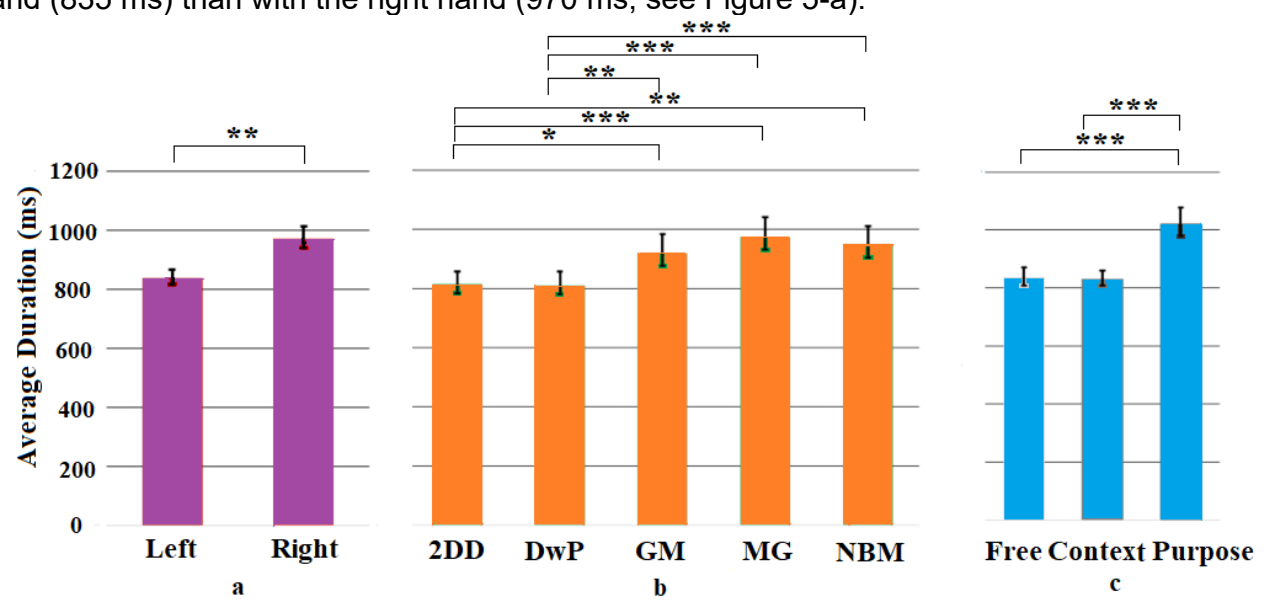
Figure 5. Fixation duration according to: a: the hand; b. the type of graphic; and c. the task. (95\% Cl). 2DD: 2D Drawings; DwP: Drawings with Perspectives; GM: Geographic Maps; MG: Mathematical Graphs; NBM: Neighborhood

$$
\text { Building Maps. }
$$

Regarding the Type of Graphic, a Pairwise Wilcox test with Bonferroni adjustment shows that there are significant differences between 2D Drawings and three other types of graphics (Geographic Maps: $p<.05$, Mathematical Graphs: $p<.001$ and Neighborhood-Building Maps: $p<.01$ ), as well as between Drawings with Perspective and three other types of graphics (Geographic Maps: $p<.01$, Mathematical Graph: $p<.001$ and Neighborhood-Building Map: $p<.001)$. Overall, fixations were shorter on 2D Drawings (813 ms) and Drawings with Perspective (810 ms) than on Geographic Maps (917 ms), Mathematical Graphs (973 ms), and Neighborhood-Building Maps (948 ms; see Figure 5-b).

Regarding the Task, a Pairwise Wilcox test with Bonferroni adjustment shows that there is a significant difference between the Free and Purpose task $(p<.001)$ and the Context and Purpose task $(p<.001)$. The average fixation duration was larger for Purpose (1019 ms) than Context (829 ms) and Free (834 ms), as illustrated in Figure 5-c.

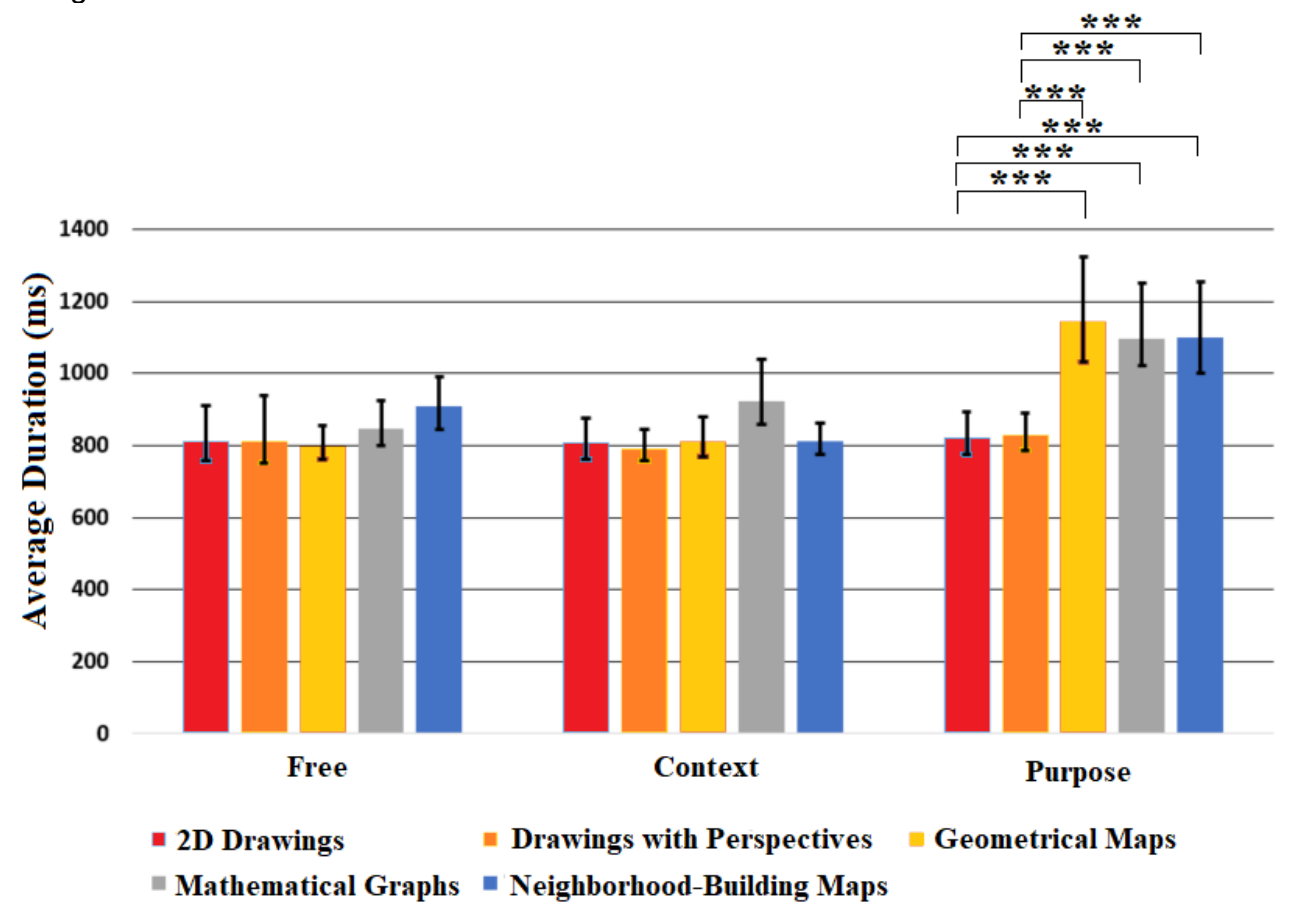

Figure 6. Average duration according to the Task and Graphic Type. $(95 \% \mathrm{Cl})$

Concerning the interaction between Task and Graphic Type $(p<.001)$, we found that: 1$)$ the aforementioned difference between 2D Drawings, Drawing with Perspective and the other types of graphic, is only true for the Purpose task (both $p<.001$ ). 2) The fixation durations for Geographic Maps, Mathematical Graphs and Neighborhood-Building Maps, are significantly longer in the Purpose task than in the two other tasks (both $p$ $<.001$; see Figure 6). 


\subsubsection{Exploration Patterns}

In our study, we started questioning the role of tactile fixations during the exploration of a whole graphic. More precisely, we looked for patterns that include successive tactile fixations. We first tagged the 296 videos with the occurrences of tactile fixations. Then we watched all the videos to tag exploration patterns relying on tactile fixations. Among the 296 videos, $98 \%$ of the videos showed two-handed exploration. This is in line with [46], which suggests that when the workspace is large enough (A3 format in our setting), there are more two-handed movements and the identification of the graphic is improved. We classified two-handed patterns including tactile fixations into the three following categories:

1) Anchor Point. In this pattern, one finger remains steady (long tactile fixation) on the anchor point while the other hand moves around the graphic (with eventual short tactile fixations) (see Figure 7). In general, the aim of this pattern is to do a comparison between graphic elements according to the anchor point. The aim is probably to create orientation and distance relations between graphic elements.

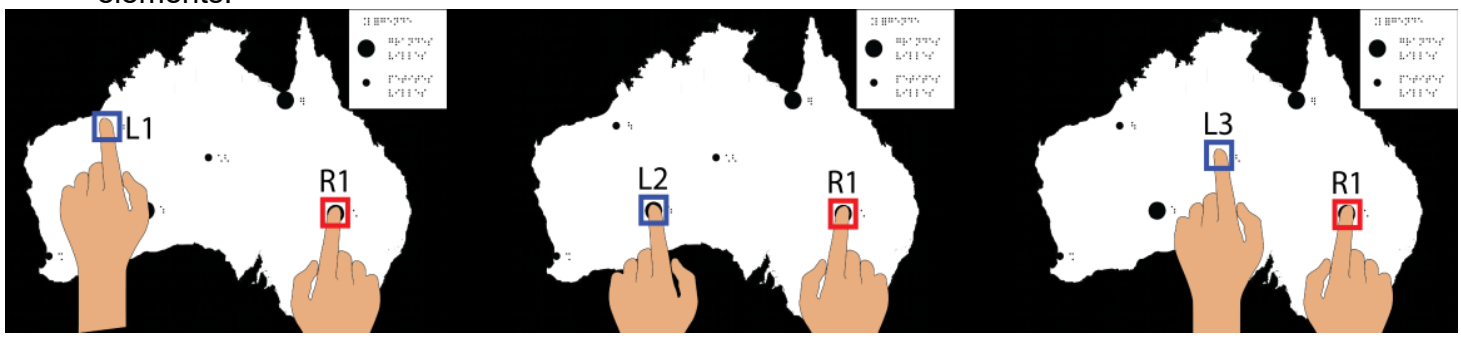

Figure 7. Conceptual illustration of the Anchor Point pattern. The right index is anchored to a point while the left index explores various other elements of the graphic.

In the Figure 8 we can identify at least one Anchor Point pattern observed with subject No. 9 exploring a geographical map in the Purpose task. It started with a long anchoring of the right finger (red square No. 21), during which the left finger performs many short fixations (blue circles No.22 to 27) at the top of the graphic.

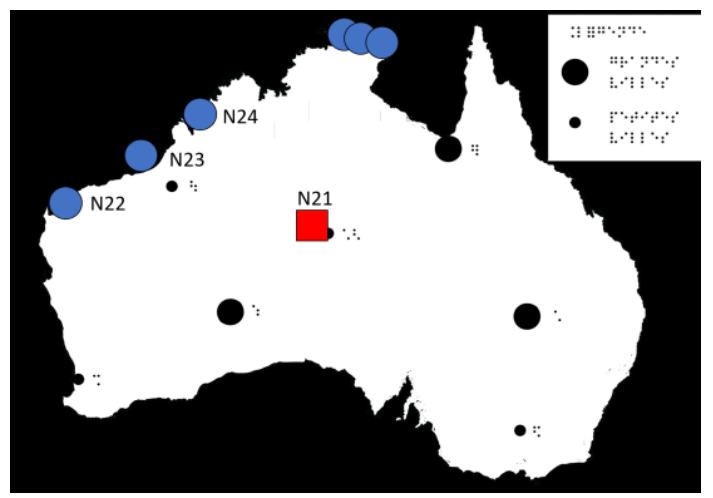

Figure 8. Example of an Anchor Point pattern. Tactile fixations with left (circles) and right (squares) hands. Numbers correspond to the tactile fixations order. Blue color for "short" fixations ( 0.5 to $1 \mathrm{~s}$ ) and red color for "long" fixations (> $5 \mathrm{~s}$ ). 
2) Switching Fingers. In this case, the user first selects a specific point as the anchor point with one hand (tactile fixation). Then he changes the finger that holds the anchor point (Figure 9). Most often the switch occurs between left and right index fingers (or vice versa).

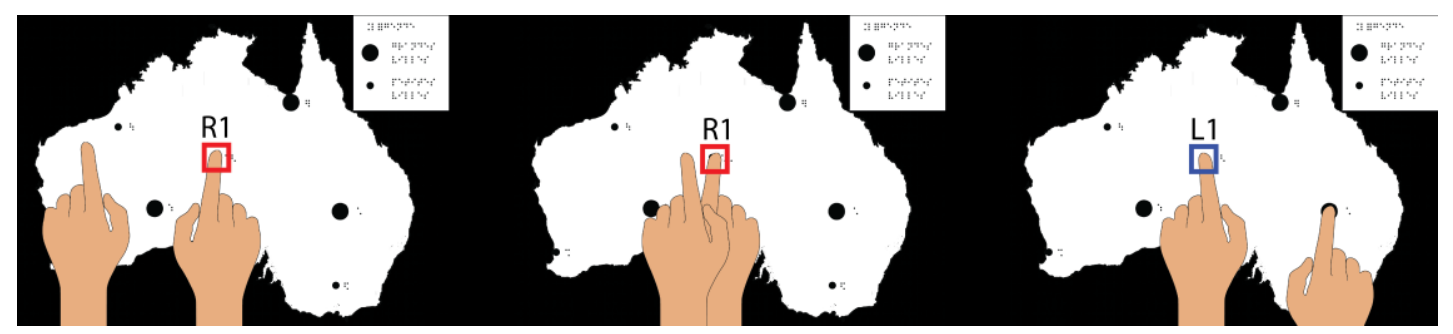

Figure 9. Conceptual illustration of Switching Fingers pattern. The right hand is in the center of the map while the left hand explores the left side of the map. After a while, the left hand replaces the right hand on its anchor point and the right hand explores the right side of the map.

Figure 10 illustrates a Switching Fingers pattern between the right and left fingers with subject No. 8 during the exploration of a geometric map in the Free task. The right finger is holding an anchor point in the middle of the graphic (orange square No. 2), during which the left finger is exploring on the left part of the graphic (fixations No. 1, 3 and 4). Then, the left finger replaces the right finger on fixation No. 5; and the right index explores the right side of the graphic (fixations No. 6 and 7 ).

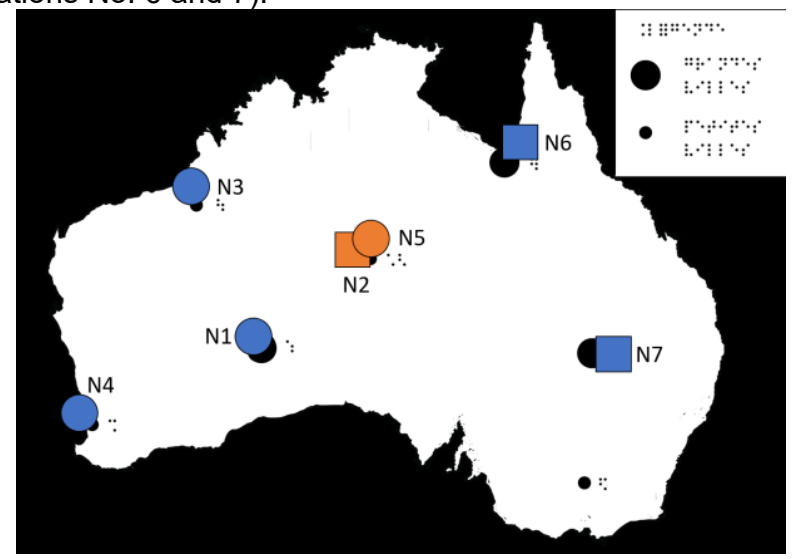

Figure 10. Example of an observed Switching Fingers pattern. Tactile fixations with left (circles) and right (squares) hands. Numbers correspond to the tactile fixations order. Blue color for "short" fixations ( 0.5 to $1 \mathrm{~s})$ and orange color for "middle" fixations ( $>1 \mathrm{~s}$ but $<5 \mathrm{~s})$.

3) Chaining Hands. This pattern appears when the right hand follows the left hand (or vice versa) from one anchor point to another one (see Figure 11). In addition, Figure 12 illustrates a Chaining Hands pattern observed with subject No. 3 during the exploration of a geometric map in task Free. In this example, the right hand (squares) follows the left hand (circles). The fixations No. 1 and 2 are the first ones (left and then right hand on the anchor point) on the left part of the graphic. Then both fingers go to the middle of the graphic (fixations No. 3 and 4). The same pattern (left and then right hand) appears 
again with fixations No. 6-7, No. 8-9 and No. 10-11. This pattern was frequently observed on points of interests in the graphics but also during Braille reading.
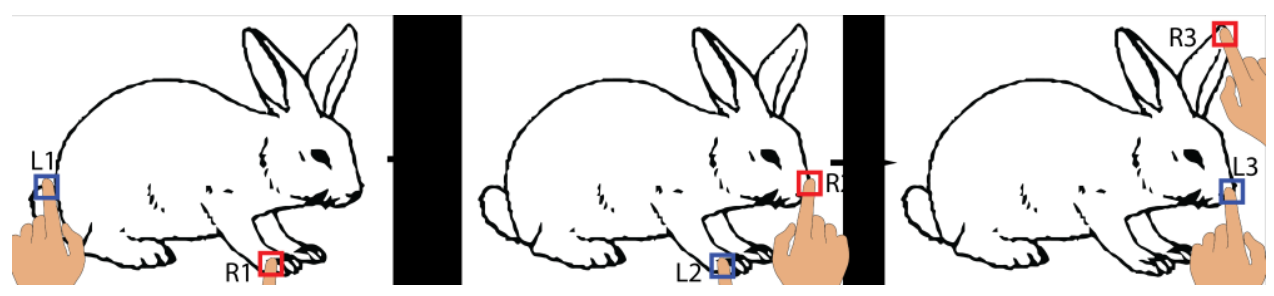

Figure 11. Conceptual illustration of Chaining Hands pattern. The right hand is on the rabbit's foot; it is joined by the left hand. The right hand goes on the muzzle of the rabbit and is followed by the left hand. The right hand goes on the ear of the rabbit, etc.

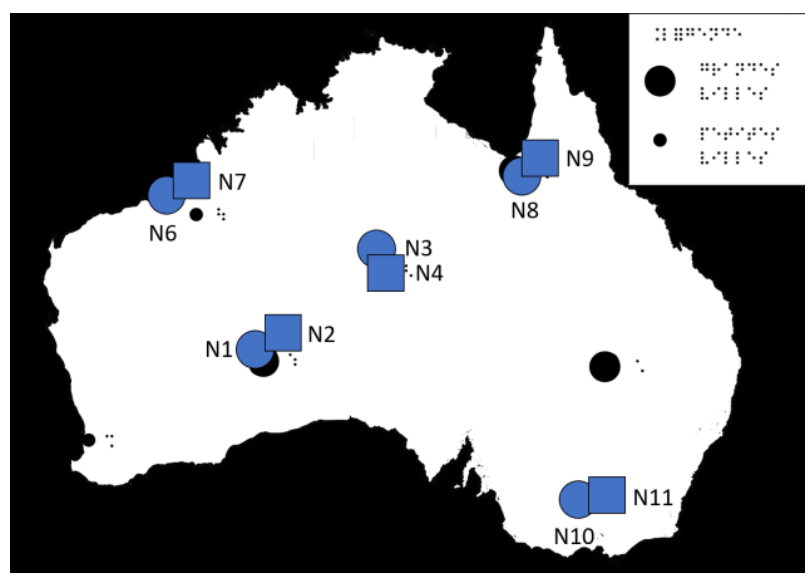

Figure 12 : Example of an observed Chaining Hands pattern. Tactile fixations with left (circles) and right (squares) hands. Numbers correspond to the tactile fixations order. Blue color for "short" fixations ( 0.5 to $1 \mathrm{~s}$ ).

\section{DISCUSSION}

In the discussion, we first answer the research questions that were introduced in this paper and then we discuss the main limitations as well as recommendations for future work.

\subsection{Can we detect tactile fixations?}

The results show that it is possible to detect tactile fixations on two-handed movement data related to tactile graphics exploration. The I-DT algorithm that we chose provides valuable results. They show that the number and duration of fixations vary according to the hand being used, the type of graphic and the task being performed. The values that are chosen for dispersion and duration in this algorithm will modify the number of "very short" fixations. Although we spent a lot of time trying to find a minimum duration threshold in the distribution of tactile fixations, we have not converged towards an indisputable value, and we finally chose a 
threshold (500 ms) that makes sense according to the tactile exploration behavior (hand movements are not as quick as eye movements). This was not prohibitive in our case because we have carried out a comparative behavioral experiment (number of fixations according to different variables). However, future work will have to ask the question about the separation between slow contour-following movements and tactile fixations on graphic elements. It could be that this threshold does not exist and that there is a continuum in tactile fixations duration (i.e. the observation of "very short" tactile fixations during smooth movements).

Our results also show that the duration of tactile fixations varies from short fixations - starting at $500 \mathrm{~ms}$ in our case - to very long fixations. The longer one was 48 seconds long, but $80 \%$ of the fixations are less than one second long. Looking at the videos, we observed that some very long fixations correspond to a "forgotten hand", which means that the subject is focusing on the other hand only. It is an open question to assess the role of what we call "forgotten hand". In the videos, we observed that these tactile fixations are less than $2 \%$. Other long fixations seem more clearly made on purpose and occur when participants are required to answer specific questions (i.e. the Purpose task in our study, see section 5.3). This observation suggests that users rely on longer fixations to answer the question because they have to find the different elements for providing the answer with the other hand, relying on the different exploration patterns that we identified. Answering specific questions requires more attention but the question is open to verify whether long fixations systematically correspond to higher cognitive loads.

\subsection{Do users perform tactile fixations with both hands?}

Both hands do tactile fixations during exploration, but, in line with [1], our results show that there are significantly more fixations with the left hand than with the right hand, independently of the graphic type or ongoing task. On the contrary the duration of tactile fixations is generally longer on the right hand than on the left hand. Among all the trials, we found 109 fixations longer than 5 seconds with the right hand, against only 46 with the left hand. This tradeoff between the number and the duration of tactile fixations is not easy to interpret but may reflect the fact that the left hand is more often used as a probe to find elements in the graphics whereas the right hand is more often used as an anchor to build topographic relationships between elements included in the graphic.

\subsection{Do tactile fixations vary according to the type of graphics and the exploration task?}

Our results show that there is a significant effect of the graphic type on the number and duration of tactile fixations. As mentioned by the tactile document maker that we consulted, 2D drawings with and without perspective are not suitable for tactile exploration. Besides, the other three categories of graphics have been designed to be interpretable for tactile exploration. One can therefore imagine that there are more areas of interest in these adapted graphics and that it is easier for users to make sense of them. This could explain the main difference in the number and duration of tactile fixations.

Another interesting result concerns the observation of significantly longer fixation durations during the "Purpose" task. This is quite logical considering that in this task the users must relate different elements of the graph to answer the question. For example, for mathematical graphs - but this is also true for the other types of graphics - they must compare several points of interest in order to deduce the correct answer to the question. It is therefore not surprising that users make longer tactile fixations on one hand while the other hand explores the points to be compared. This is probably even more true if the number of comparisons needed to answer the question being asked increases, but this should be addressed in future work. 


\subsection{Can we relate tactile fixations to exploration strategies?}

Our results are in line with previous observations on exploration strategies showing that both hands are frequently doing stops as well as back and forth movements when exploring a tactile graphic [22] [43]. They suggest that tactile fixations are gathered into patterns which themselves can be grouped in time and space to build more elaborate cognitive strategies aiming to interpret the graphic. For example, the "point of reference" strategy mentioned by [43] is an assembly of Anchor Point patterns that allows the user to understand the relationships between an important element of the graphic and several other elements around it. The Switching Fingers pattern seems to be an adaptation of the Anchor Point pattern, which avoids crossing hands when exploring both sides of the graphic. In other words, both patterns would give evidence of the use of the same cognitive strategy but considering the constraint of the spatial layout of the graphic. During the Chaining Hands pattern, participants browse a series of elements of the configuration. This pattern can give rise to the cyclic cognitive strategy observed by [15]. It would reveal the intention to memorize a "route" or a succession of meaningful elements in the graphic. In addition, although the Anchor Point pattern has already been observed by Wijntjes et al. [46], the Switching Fingers and Chaining Hands patterns have never been observed before. We suggest that these two-handed exploration patterns are at an intermediate level and can be put together to give rise to higher level successful strategies that have been previously described (e.g. the reference point and cyclic strategies respectively observed in [43] and [15]).

\subsection{Lessons learned and implications for $\mathrm{HCl}$ and other fields}

The main contribution of our study is a method to track fingers movements and then to detect tactile fixations, which can be further used to assess the non-visual graphics exploration strategies involved. This method can have many implications for specialized teachers, document makers, designers and researchers. We detail different implications below:

1) To our knowledge, there are instruction techniques related to how to explore tactile graphics at a higher level in special education for people with $\mathrm{VI}$ (start with reading the title, finding and exploring the legend, etc.). However, there is no recognized instruction technique relying directly on tactile fixations or exploration patterns based on tactile fixations. With our method, special education teachers can identify tactile fixations and exploration patterns leading to a better understanding of tactile drawings. They can then propose recommendations on how to better perform two-handed exploration of tactile drawings.

2) Tactile document makers can easily observe that some important points of tactile graphics have not been touched or, on the contrary, that irrelevant points of the drawings were touched many times with long tactile fixations, which may reflect user's confusion. They can then propose guidelines to better design tactile drawings.

3) Interaction designers can design feedback for audio-tactile drawings (see [7]) that is coherent with the user's behavior. For instance, during the discovery phase of a tactile drawing, there is no need to design feedback for short tactile fixations because they are too frequent, but one can add feedback on long fixations to assist the user's exploration (e.g. naming the point). In addition, the system can inform users that some important points of the drawings have not been touched yet and indicate where they are. During the memorization phase (i.e. after the discovery phase), command menus can be added to relevant points of the drawing (points with long fixations) to better compare or memorize the relative 
location of points of interest, and hence improve the comprehension of the drawing. Interestingly, these contextual menus will not interrupt the exploration process.

4) Finally, researchers in psychology and $\mathrm{HCl}$ can use this method to better understand the exploration strategies of tactile drawing. Many questions related to the link between the observed behaviors, the involved cognitive strategies and the participants' performances can be addressed.

\subsection{Limitations and future work}

There are limitations and open questions arising from this initial study on Tactile Fixations. The first limitation we can mention concerns the technical implementation of the Tactile Fixation detection algorithm. We opted for a visual tracking based on colors. Although this is easy to implement, illumination conditions and occlusions induce tracking breakdowns that we corrected by visually checking the videos (with our finger tracking algorithm, the average tracking loss rate was less than $5 \%$ ). This is time consuming and not optimal. It would be interesting to use a more reliable tracking system. An infra-red motion capture system could ease tracking in lab conditions, but only if the sensors placed on the fingers do not hinder exploration. In terms of interpretation, our results need to be complemented by further analysis of the occurrences of exploration patterns and their concatenation to build more elaborate cognitive strategies. Beyond the detection of tactile fixations, it would be interesting to implement algorithms to detect exploration patterns in order to finally link them to cognitive strategies. Besides, we did not investigate the potential impact of tactile expertise on the fixations. Finally, thanks to our experimental design including three tasks (free, context, purpose), we have observed the differences in exploration behaviors according to the task being performed. This result confirms the hypothesis that we made by observing people with visual impairments exploring tactile graphics under different uncontrolled conditions. It opens up a set of research questions related to the impact of the task on exploration behaviors for which our tracking algorithm will be highly relevant.

We also think about interesting future work based on Tactile Fixations. The concept of tactile fixations comes from research studies on eye fixations. In this domain, fixations can indicate the most salient elements in the visual scene, but they can also indicate the attention paid to certain elements in the scene [23]. Similarly, tactile fixations can be used to identify the salient elements of the tactile graphic, but also the elements - salient or not - on which the user is focusing his/her attention. As mentioned in the previous section, these analyses can lead to design better tactile graphics, inducing less difficulties during exploration, and they can also inform the design of more usable interaction techniques with hybrid or digital graphics [11]. For example, one can imagine that command menus appear as a function of the time spent exploring certain elements of the graph or when certain behavioral patterns - such as the Anchor Point - are detected. Such a system could help young users with visual impairments, who are learning to read tactile graphics, to interpret them more easily.

\section{CONCLUSION}

In this work, we proposed a behavioral marker that we called "tactile fixation", which can help to understand how people with VI explore raised-line graphics. The results from analyzing a large data set of finger explorations, collected through a controlled study with participants with $\mathrm{VI}$, demonstrate that such fixations not only exist, but also can help to explain the different behaviors observed during the exploration of tactile graphics. We are convinced that future research on tactile fixations can provide valuable insights on how to design better tactile graphics but also on the difficulties observed during the exploration of tactile documents by people with 
visual impairments. We also think that it can inform the design of more usable interaction techniques helping to explore and understand non-visual digital graphics. By introducing such a novel marker, we hope that our work will inform future research and open new research questions with the common goal of improving the accessibility of graphics to people with VI.

\section{ACKNOWLEDGMENTS}

We thank all the participants, the laboratory "Cherchons pour Voir" and China Scholarship Council.

\section{REFERENCES}

[1] Sandra Bardot, Marcos Serrano, Bernard Oriola, and Christophe Jouffrais. 2017. Identifying how Visually Impaired People Explore Raised-line Diagrams to Improve the Design of Touch Interfaces. In Proceedings of the $2017 \mathrm{CHI}$ Conference on Human Factors in Computing Systems (CHI '17). Association for Computing Machinery, New York, NY, USA, $550-555$. https://doi.org/10.1145/3025453.3025582

[2] Sandra Bardot, Marcos Serrano, Simon Perrault, Shengdong Zhao, and Christophe Jouffrais. 2019. Investigating Feedback for TwoHanded Exploration of Digital Maps Without Vision. In IFIP Conference on Human-Computer Interaction (INTERACT 2019), $305-324$. https://doi.org/10.1007/978-3-030-29381-9 19

[3] B L Bentzen. 1983. Tactile specifications of route configurations. In Proceedings of the First International Symposium on Maps and Graphics for the Visually Handicapped. Washington, DC: Association of American Geographers.

[4] David Beymer, Peter Z. Orton, and Daniel M. Russell. 2007. An eye tracking study of how pictures influence online reading. In IFIP Conference on Human-computer Interaction (INTERACT 2007). Springer-Verlag, Berlin, Heidelberg, $456-460$. https://doi.org/10.1007/978-3-540-74800-7 41

[5] Tanja Blascheck, Lonni Besançon, Anastasia Bezerianos, Bongshin Lee, Petra Isenberg. Glanceable Visualization: Studies of Data Comparison Performance on Smartwatches. IEEE Transactions on Visualization and Computer Graphics, Institute of Electrical and Electronics Engineers, 2019, 25 (1), pp. 616-629.

[6] Anke Brock, Samuel Lebaz, Bernard Oriola, Delphine Picard, Christophe Jouffrais, and Philippe Truillet. 2012. Kin'touch: understanding how visually impaired people explore tactile maps. In $\mathrm{CHI}$ '12 Extended Abstracts on Human Factors in Computing Systems (CHI EA '12). Association for Computing Machinery, New York, NY, USA, 2471-2476. DOI: https://doi.org/10.1145/2212776.2223821

[7] Anke Brock, Philippe Truillet, Bernard Oriola, Delphine Picard, and Christophe Jouffrais. 2015. Interactivity improves usability of geographic maps for visually impaired people. Human-Computer Interaction, 30, 2: 156-194.

[8] Emeline Brulé, Brianna J. Tomlinson, Oussama Metatla, Christophe Jouffrais, and Marcos Serrano. 2020. Review of Quantitative Empirical Evaluations of Technology for People with Visual Impairments. In Proceedings of the $2020 \mathrm{CHI}$ Conference on Human Factors in Computing Systems (CHI '20). Association for Computing Machinery, New York, NY, USA, 1-14. DOI: https://doi.org/10.1145/3313831.3376749

[9] Georg Buscher, Edward Cutrell, and Meredith Ringel Morris. 2009. What do you see when you're surfing? using eye tracking to predict salient regions of web pages. In Proceedings of the SIGCHI Conference on Human Factors in Computing Systems (CHI '09). ACM, New York, NY, USA, 21-30. DOI: https://doi.org/10.1145/1518701.1518705

[10] Julie Ducasse, Marc J-M Macé, Marcos Serrano, and Christophe Jouffrais. 2016. Tangible Reels: Construction and Exploration of Tangible Maps by Visually Impaired Users. In Proceedings of the $2016 \mathrm{CHI}$ Conference on Human Factors in Computing Systems (CHI '16). Association for Computing Machinery, New York, NY, USA, 2186-2197. https://doi.org/10.1145/2858036.2858058

[11] Julie Ducasse, Anke M. Brock and Christophe Jouffrais. 2018. Accessible interactive maps for visually impaired users. In Mobility of visually impaired people, pp. 537-584. Springer, Cham.

[12] Polly Edman. 1992. Tactile graphics. American Foundation for the Blind.

[13] Yvonne Eriksson, Gunnar Jansson, and Monica Strucel. 2003. Tactile maps: guidelines for the production of maps for the visually impaired. Swedish Braille Authority, Swedish Library of Talking Books and Braille, (TPB).

[14] Yvonne Eriksson and Monica Strucel. 1995. Production of tactile graphics on swellpaper. Swedish Library of Talking Books and Braille.

[15] Florence Gaunet and Catherine Thinus-Blanc. 1996. Early-blind subjects' spatial abilities in the locomotor space: Exploratory strategies and reaction-to-change performance. Perception 25, 8: 967-981.

[16] Timo Götzelmann and Aleksander Pavkovic. 2014. Towards automatically generated tactile detail maps by 3D printers for blind persons. In Proceedings of International Conference on Computers for Handicapped Persons (ICCHP 2014), pp. 1-7, Springer, Cham.

[17] William Grussenmeyer, Jesel Garcia, and Fang Jiang. 2016. Feasibility of using haptic directions through maps with a tablet and smart watch for people who are blind and visually impaired. In Proceedings of the 18th International Conference on Human-Computer Interaction with Mobile Devices and Services (MobileHCl '16), Association for Computing Machinery, New York, NY, USA, 83-89. https://doi.org/10.1145/2935334.2935367 
[18] Tiago Guerreiro, Kyle Montague, João Guerreiro, Rafael Nunes, Hugo Nicolau, and Daniel J.V. Gonçalves. 2015. Blind People Interacting with Large Touch Surfaces: Strategies for One-handed and Two-handed Exploration. In Proceedings of the 2015 International Conference on Interactive Tabletops \& Surfaces (ITS '15). Association for Computing Machinery, New York, NY, USA, $25-34$. https://doi.org/10.1145/2817721.2817743

[19] Yvette Hatwell, Arlette Streri and Edouard Gentaz. 2003. Touching for knowing: cognitive psychology of haptic manual perception. Vol 53. John Benjamins Publishing.

[20] Morton A. Heller, Melissa McCarthy, and Ashley Clark. 2005. Pattern perception and pictures for the blind. Psicologica 26, $1: 161-171$.

[21] Morton A Heller. 1989. Picture and Pattern Perception in the Sighted and the Blind: The Advantage of the Late Blind. Perception 18, 3: 379-389. https://doi.org/10.1068/p180379

[22] Everett W. Hill, John J. Rieser, M-M. Hill, Marc Hill, J. Halpin, and R. Halpin. 1993. How persons with visual impairments explore novel spaces: Strategies of good and poor performers. Journal of visual impairment \& blindness 87, 8: 295-301.

[23] James E. Hoffman. 1998. Visual attention and eye movements. Attention, 31: 119-153.

[24] Leona Holloway, Kim Marriott, and Matthew Butler. 2018. Accessible Maps for the Blind: Comparing 3D Models with Tactile Graphics. In Proceedings of the $2018 \mathrm{CHI}$ Conference on Human Factors in Computing Systems (CHI '18). Association for Computing Machinery, New York, NY, USA, 1-13. https://doi.org/10.1145/3173574.3173772

[25] R.Dan Jacobson. 1998. Cognitive mapping without sight: Four preliminary studies of spatial learning. Journal of Environmental Psychology. 18, 3: 289-305

[26] Shaun K. Kane, Meredith Ringel Morris, Annuska Z. Perkins, Daniel Wigdor, Richard E. Ladner, Jacob O. Wobbrock. 2011. Access Overlays: Improving Non-Visual Access to Large Touch Screens for Blind Users. In Proceedings of the 24th annual ACM symposium on User interface software and technology (UIST '11). Association for Computing Machinery, New York, NY, USA, $273-282$. https://doi.org/10.1145/2047196.2047232

[27] John M. Kennedy and Juan Bai. 2002. Haptic Pictures: Fit Judgments Predict Identification, Recognition Memory, and Confidence. Perception 31, 8: 1013-1026. https://doi.org/10.1068/p3259

[28] Roberta L. Klatzky, Jack M. Loomis, Susan J. Lederman, Hiromi Wake, and Naofumi Fujita. 1993. Haptic identification of objects and their depictions. Perception \& Psychophysics 54, 2: 170-178. https://doi.org/10.3758/BF03211752

[29] Orly Lahav and David Mioduser. 2008. Haptic-feedback support for cognitive mapping of unknown spaces by people who are blind. International Journal of Human-Computer Studies 66, 1: 23-35.

[30] Steven Landau, Michael Russell, Karen Gourgey, Jane N. Erin and Jennifer Cowan. Use of the talking tablet in mathematics testing. 2003. Journal of Visual Impairment \& Blindness, 97, 2: 85-96.

[31] Samuel Lebaz, Christophe Jouffrais, and Delphine Picard. 2012. Haptic identification of raised-line drawings: high visuospatial imagers outperform low visuospatial imagers. Psychological research 76, 5: 667-675. https://doi.org/10.1007/s00426-011-0351-6

[32] Susan J. Lederman and Roberta L. Klatzky, April Collions, and Jackie Wardell. 1987. Exploring Environments by Hand or Foot: TimeBased Heuristics for Encoding Distance in Movement Space. Journal of Experimental Psychology: Learning, Memory, and Cognition 13, 4: 606. https://doi.org/10.1037/0278-7393.13.4.606

[33] Susan J. Lederman and Roberta L. Klatzky. 2009. Haptic perception: A tutorial. Attention, Perception \& Psychophysics 71,7 : 14391459. https://doi.org/10.3758/APP.71.7.1439

[34] Susan J. Lederman and Roberta L. Klatzky. 1987. Hand movements: A window into haptic object recognition. Cognitive psychology 19, 3: $342-368$

[35] Susan J. Lederman, Roberta L. Klatzky, Cynthia Chataway, and Craig D. Summers. 1990. Visual mediation and the haptic recognition of two-dimensional pictures of common objects. Perception \& Psychophysics 47, 1: 54-64. https://doi.org/10.3758/BF03208164

[36] Jack M. Loomis, Roberta L. Klatzky, and Susan J. Lederman. 1991. Similarity of tactual and visual picture recognition with limited field of view. Perception 20, 2: 167-177.

[37] Irene Miller, Aquinas Pather, Janet Milbury, Lucia Hasty, Allison O’Day, Diane Spence, and S. Osterhaus. 2010. Guidelines and Standards for Tactile Graphics. The Braille Authority of North America.

[38] Carson Y. Nolan and June E. Morris. 1971. Improvement of Tactual Symbols for Blind Children. Final Report. 88.

[39] R.M. Sakia. The Box-Cox transformation technique: a review. Journal of the Royal Statistical Society: Series D (The Statistician), 41 (2), 169-178.

[40] Dario D. Salvucci and Joseph H. Goldberg. 2000. Identifying fixations and saccades in eye-tracking protocols. In Proceedings of the symposium on eye tracking research \& applications (ETRA '00), 71-78. https://doi.org/10.1145/355017.355028

[41] Joan G. Snodgrass and Mary Vanderwart. 1980. A standardized set of 260 pictures: norms for name agreement, image agreement, familiarity, and visual complexity. Journal of experimental psychology: Human learning and memory 6, 2: 174-215.

[42] Mark Symmons and Barry Richardson. 2000. Raised line drawings are spontaneously explored with a single finger. Perception 29, 5: $621-626$

[43] Jon M. Tellevik. 1992. Influence of spatial exploration patterns on cognitive mapping by blindfolded sighted persons. Journal of Visual Impairment \& Blindness, 86, 5: 221-224.

[44] Edward C. Tolman. 1948. Cognitive maps in rats and men. Psychological review 55, 4: 189.

[45] Leanne J. Thompson, Edward P. Chronicle, and Alan F. Collins. 2006. Enhancing 2-D Tactile Picture Design from Knowledge of 3-D 
Haptic Object Recognition. European Psychologist 11, 2: 110-118. https://doi.org/10.1027/1016-9040.11.2.110

[46] Maarten WA Wijntjes, Thijs Van Lienen, Ilse M. Verstijnen, and Astrid M L Kappers. 2008. The influence of picture size on recognition and exploratory behaviour in raised-line drawings. Perception 37, 4: 602-614. https://doi.org/10.1068/p5714

[47] Kaixing Zhao, Marcos Serrano, Bernard Oriola, and Christophe Jouffrais. 2020. VibHand: On-Hand Vibrotactile Interface Enhancing NonVisual Exploration of Digital Graphics. Proc. ACM Hum.-Comput. Interact. 4, ISS, Article 207 (November 2020), 19 pages. DOI: https://doi.org/10.1145/3427335 